\title{
CHAPTER 2 OF RAMANUJAN'S SECOND NOTEBOOK
}

\author{
by BRUCE C. BERNDT $\dagger$, PADMINI T. JOSHI, and B. M. WILSON
}

(Received 12 February, 1980)

When Ramanujan died in 1920 he left behind three notebooks containing statements of a few thousand theorems, mostly without proofs. The second notebook is an enlarged edition of the first, and the third is short and fragmentary. Thus our primary attention may be directed toward the second notebook. In the decade following Ramanujan's death, G. $\mathrm{N}$. Watson and B. M. Wilson agreed to perform the enormous task of editing the notebooks. Unfortunately, this task was never completed, possibly, in part, due to the premature death of Wilson in 1935. In 1957, a photostat edition [19] of the notebooks was published, but no editing whatsoever was undertaken.

After Watson died in 1965, his papers were donated by Mrs. Watson to the Trinity College library at Cambridge University at the suggestion of R. A. Rankin. Included in these manuscripts was the unfinished work that Watson and Wilson accomplished in their efforts to edit the notebooks. For many chapters, a substantial amount of work had been done, but some chapters were virtually untouched. Wilson was given the task of editing the earlier chapters in the second notebook, and Chapter 2 was almost completed by Wilson. The first two named authors have taken Wilson's notes for this chapter and completed the work. It is hoped that other chapters will be similarly edited. Before the first author had learned of the existence of the Watson-Wilson manuscripts at Trinity college, he had edited Chapter 14 [4]. (Watson examined only six entries from Chapter 14.)

Chapter 2 is fairly elementary, but several of the formulae are very intriguing and evince Ramanujan's ingenuity and cleverness. Ramanujan gives more proofs in this chapter than in most of the later chapters. Chapter 2 contains 68 theorems and formulas, most of which are identities between finite sums. Many of these identities involve $\arctan x$, and because this function arises so frequently in the sequel, we shall put $A(x)=\arctan x$. It will be assumed that $-\pi / 2 \leq A(x) \leq \pi / 2$. Several of Ramanujan's theorems concerning this function arise from the elementary equalities

$$
A(x)+A(y)=A\left(\frac{x+y}{1-x y}\right)
$$

except when $x y>1$, and

$$
A(x)-A(y)=A\left(\frac{x-y}{1+x y}\right)
$$

except when $-x y>1$.

$\nmid$ Research partially supported by National Science Foundation grant no. MCS-7903359.

Glasgow Math. J. 22 (1981) 199-216. 
Entries $1,2,4,5$, and 6 involve the function

$$
\varphi(a, n)=1+2 \sum_{k=1}^{n} \frac{1}{(a k)^{3}-a k},
$$

where $a$ is an integer exceeding one. Furthermore, we put $\varphi(a)=\lim _{n \rightarrow \infty} \varphi(a, n)$.

ENTRY 1. For each positive integer $n$,

$$
\sum_{k=1}^{n} \frac{1}{n+k}=\frac{n}{2 n+1}+\sum_{k=1}^{n} \frac{1}{(2 k)^{3}-2 k}
$$

Proof. We give Ramanujan's proof. In the easily verified identity

$$
\frac{1}{x^{3}-x}=\frac{1}{2(x-1)}+\frac{1}{2(x+1)}-\frac{1}{x}
$$

let $x=2 k$ and sum on $k, 1 \leq k \leq n$. The right side of (1.1) is then found to be equal to

$$
\begin{aligned}
\frac{1}{2} \sum_{k=1}^{n} \frac{1}{2 k-1}+\frac{1}{2} \sum_{k=1}^{n} \frac{1}{2 k+1}-\frac{1}{2} \sum_{k=1}^{n} \frac{1}{k}+\frac{n}{2 n+1} & =\sum_{k=1}^{n} \frac{1}{2 k-1}-\frac{1}{2} \sum_{k=1}^{n} \frac{1}{k} \\
& =\sum_{k=1}^{2 n} \frac{1}{k}-\sum_{k=1}^{n} \frac{1}{k}=\sum_{k=1}^{n} \frac{1}{n+k} .
\end{aligned}
$$

COROLLARY. $\log 2=\frac{1}{2} \varphi(2)$.

Proof. Since [2, p. 43],

$$
\lim _{x \rightarrow \infty}\left\{\sum_{k \leq x} \frac{1}{k}-\log x\right\}=\gamma
$$

where $\gamma$ denotes Euler's constant, we find from the last equality in (1.3) that

$$
\lim _{n \rightarrow \infty} \sum_{k=1}^{n} \frac{1}{n+k}=\lim _{n \rightarrow \infty}\left\{\left(\sum_{k=1}^{2 n} \frac{1}{k}-\log (2 n)\right)-\left(\sum_{k=1}^{n} \frac{1}{k}-\log n\right)\right\}+\log 2=\log 2 .
$$

The result now follows from Entry 1 and the definition of $\varphi(a)$.

There is a different proof of this corollary in Ramanujan's first notebook [19, vol. I., p. 7]. This proof is also discussed in [3, p. 154].

EXAMPLE. For each positive integer $n$,

$$
\sum_{k=1}^{n} \frac{n-k}{n+k}=2 n \sum_{k=1}^{n} \frac{1}{(2 k-1) 2 k(2 k+1)}-\frac{n}{2 n+1} .
$$

Proof. The proof below is given by Ramanujan. Multiply both sides of (1.1) by $2 n$ to get

$$
\sum_{k=1}^{n} \frac{2 n}{n+k}=2 n \sum_{k=1}^{n} \frac{1}{(2 k-1) 2 k(2 k+1)}+\frac{2 n^{2}}{2 n+1}
$$


Subtract 1 from each term on the left side of (1.5) and $n$ from the right side of (1.5) to achieve the desired equality.

ENTRY 2. For each positive integer $n$,

$$
\sum_{k=1}^{2 n+1} \frac{1}{n+k}=\varphi(3, n)
$$

Proof. Using (1.2), we find that

$$
\varphi(3, n)=1+2 \sum_{k=1}^{n}\left\{\frac{1}{2(3 k-1)}+\frac{1}{2(3 k+1)}-\frac{1}{3 k}\right\}=\sum_{k=1}^{3 n+1} \frac{1}{k}-\sum_{k=1}^{n} \frac{1}{k},
$$

from which the desired result follows.

COROLlaRY. $\log 3=\varphi(3)$.

Proof. The proof is like that of the corollary to Entry 1. Let $n \rightarrow \infty$ in (2.1) and use the fact that, by (1.4),

$$
\lim _{n \rightarrow \infty} \sum_{k=n+1}^{3 n+1} \frac{1}{k}=\log 3
$$

Ramanujan's proof of the corollary above is similar to the aforementioned proof that he gave in the first notebook for the corollary of Entry 1. Ramanujan replaced $n$ by $1 / d x$ in the left side of (2.1) and regarded this sum as a Riemann sum. Thus,

$$
\lim _{d x \rightarrow 0} \sum_{k=1}^{2 n+1} \frac{d x}{1+k d x}=\int_{1}^{3} \frac{d x}{x}=\log 3,
$$

from which the corollary follows.

ENTRY 3. For each positive integer $n$,

$$
\sum_{k=1}^{2 n+1} A\left(\frac{1}{n+k}\right)=\frac{\pi}{4}+\sum_{k=1}^{n} A\left(\frac{10 k}{\left(3 k^{2}+2\right)\left(9 k^{2}-1\right)}\right) .
$$

Proof. By (0.1) and (0.2), respectively,

$$
A\left(\frac{1}{3 k-1}\right)+A\left(\frac{1}{3 k+1}\right)=A\left(\frac{6 k}{9 k^{2}-2}\right)
$$

and

$$
A\left(\frac{1}{k}\right)-A\left(\frac{1}{3 k}\right)=A\left(\frac{2 k}{3 k^{2}+1}\right)
$$

for each positive integer $k$. By (3.2), (3.3), and (0.2), we find that

$$
A\left(\frac{1}{3 k-1}\right)+A\left(\frac{1}{3 k}\right)+A\left(\frac{1}{3 k+1}\right)-A\left(\frac{1}{k}\right)=A\left(\frac{10 k}{\left(3 k^{2}+2\right)\left(9 k^{2}-1\right)}\right) \text {. }
$$


If we now sum both sides of (3.4) for $1 \leq k \leq n$, we readily complete the proof of Entry 3 .

Note that, by (3.1) and Taylor's theorem,

$$
\begin{aligned}
\sum_{k=1}^{n} A\left(\frac{10 k}{\left(3 k^{2}+2\right)\left(9 k^{2}-1\right)}\right) & =\sum_{k=n+1}^{3 n+1}\left\{\frac{1}{k}+O\left(\frac{1}{k^{3}}\right)\right\}-\frac{\pi}{4} \\
& =\sum_{k=n+1}^{3 n+1} \frac{1}{k}+O\left(\frac{1}{n^{2}}\right)-\frac{\pi}{4} .
\end{aligned}
$$

Letting $n \rightarrow \infty$ and using (2.2), we deduce that

$$
\sum_{k=1}^{\infty} A\left(\frac{10 k}{\left(3 k^{2}+2\right)\left(9 k^{2}-1\right)}\right)=\log 3-\frac{\pi}{4},
$$

which is given by Ramanujan in his first notebook [19, vol. I, p. 9].

ENTRY 4. For each positive integer $n$,

$$
\sum_{k=1}^{n} \frac{1}{n+k}+\sum_{k=0}^{n} \frac{1}{2 n+2 k+1}=\varphi(4, n)=\sum_{k=1}^{4 n+1} \frac{(-1)^{k+1}}{k}+\frac{1}{2} \sum_{k=1}^{2 n} \frac{(-1)^{k+1}}{k} .
$$

Proof. The complete proof is given by Ramanujan. By (1.2),

$$
\begin{aligned}
\varphi(4, n) & =1+\sum_{k=1}^{n}\left\{\frac{1}{4 k-1}+\frac{1}{4 k+1}-\frac{1}{2 k}\right\} \\
& =\sum_{k=1}^{4 n+1} \frac{1}{k}-\frac{1}{2} \sum_{k=1}^{2 n} \frac{1}{k}-\frac{1}{2} \sum_{k=1}^{n} \frac{1}{k} \\
& =\sum_{k=1}^{3 n+1} \frac{1}{n+k}-\sum_{k=1}^{n} \frac{1}{2 n+2 k} \\
& =\sum_{k=1}^{n} \frac{1}{n+k}+\sum_{k=0}^{n} \frac{1}{2 n+2 k+1},
\end{aligned}
$$

which proves the first equality in (4.1).

Next, using the second equality in (4.2), we find that

$$
\begin{aligned}
\varphi(4, n) & =\sum_{k=1}^{4 n+1} \frac{1}{k}-2 \sum_{k=1}^{2 n} \frac{1}{2 k}+\frac{1}{2} \sum_{k=1}^{2 n} \frac{1}{k}-\sum_{k=1}^{n} \frac{1}{2 k} \\
& =\sum_{k=1}^{4 n+1} \frac{(-1)^{k+1}}{k}+\frac{1}{2} \sum_{k=1}^{2 n} \frac{(-1)^{k+1}}{k},
\end{aligned}
$$

which establishes the second equality in (4.1).

In the proof above, and elsewhere, Ramanujan frequently used a rather unorthodox notation. Thus, for example, $\sum \frac{1}{2 n}$ means $\sum_{k=1}^{2 n} \frac{1}{k}$ and $\sum \frac{1}{4 n+1}$ means $\sum_{k=1}^{4 n+1} \frac{1}{k}$. 
The corollary below represents the first problem that Ramanujan submitted to the Journal of the Indian Mathematical Society [16], [18, p. 322]. Ironically, this result was previously posed as a problem by Lionnet [12] in 1879 . The problem and its solution are also given in Chrystal's textbook [6, p. 249].

COROLLARY. $\frac{3}{2} \log 2=\varphi(4)$.

Proof. Let $n \rightarrow \infty$ on the right side of (4.1) and use the equality $\sum_{k=1}^{\infty}(-1)^{k+1} / k=$ $\log 2$.

ENTRY 5. For each positive integer $n$,

$$
\varphi(6, n)=\frac{2}{3} \sum_{k=1}^{n} \frac{1}{n+k}+\sum_{k=0}^{2 n} \frac{1}{2 n+2 k+1} .
$$

Proof. By (1.2),

$$
\begin{aligned}
\varphi(6, n) & =1+\sum_{k=1}^{n}\left\{\frac{1}{6 k-1}+\frac{1}{6 k+1}-\frac{1}{3 k}\right\} \\
& =\sum_{k=1}^{6 n+1} \frac{1}{k}-\frac{1}{3} \sum_{k=1}^{n} \frac{1}{k}-\sum_{k=1}^{n}\left\{\frac{1}{6 k-4}+\frac{1}{6 k-3}+\frac{1}{6 k-2}+\frac{1}{6 k}\right\} \\
& =\sum_{k=1}^{6 n+1} \frac{1}{k}-\frac{1}{3} \sum_{k=1}^{n} \frac{1}{k}-\frac{1}{2} \sum_{k=1}^{3 n} \frac{1}{k}-\frac{1}{3} \sum_{k=1}^{n} \frac{1}{2 k-1} \\
& =\sum_{k=1}^{6 n+1} \frac{1}{k}-\frac{1}{3} \sum_{k=1}^{n} \frac{1}{k}-\frac{1}{2} \sum_{k=1}^{3 n} \frac{1}{k}-\frac{1}{3}\left\{\sum_{k=1}^{2 n} \frac{1}{k}-\frac{1}{2} \sum_{k=1}^{n} \frac{1}{k}\right\} \\
& =\sum_{k=1}^{6 n+1} \frac{1}{k}-\frac{1}{2} \sum_{k=1}^{3 n} \frac{1}{k}-\frac{1}{3} \sum_{k=1}^{2 n} \frac{1}{k}-\frac{1}{6} \sum_{k=1}^{n} \frac{1}{k} \\
& =\sum_{k=n+1}^{6 n+1} \frac{1}{k}-\frac{1}{2} \sum_{k=n+1}^{3 n} \frac{1}{k}-\frac{1}{3} \sum_{k=n+1}^{2 n} \frac{1}{k} \\
& =\sum_{k=n+1}^{2 n} \frac{1}{k}+\sum_{k=n}^{3 n} \frac{1}{2 k+1}-\frac{1}{3} \sum_{k=n+1}^{2 n} \frac{1}{k},
\end{aligned}
$$

from which (5.1) easily follows.

COROLLARY. $\frac{1}{2} \log 3+\frac{1}{3} \log 4=\varphi(6)$.

Proof. Letting $n \rightarrow \infty$ in (5.1) and employing (1.4), we achieve the desired equality.

EXAMPLE 1. $\frac{1}{4} \log 2=\sum_{k=1}^{\infty} \frac{1}{\{2(2 k-1)\}^{3}-2(2 k-1)}$.

Proof. The right side above is equal to $\frac{1}{2}\{\varphi(2)-\varphi(4)\}$. Hence, the result follows from the corollaries to Entries 1 and 4. 
EXAMPLE 2. $\log 2=1+\sum_{k=1}^{\infty} \frac{2(-1)^{k}}{(2 k)^{3}-2 k}$.

Proof. The right side above is $2 \varphi(4)-\varphi(2)$, and so the example again follows from the corollaries to Entries 1 and 4.

EXAMPLE 3. For each positive integer $n$,

$$
2 \varphi(4, n)=\varphi(2,2 n)+\frac{1}{2} \varphi(2, n)+\frac{1}{(4 n+1)(4 n+2)}
$$

Proof. From Entries 1 and 4, respectively,

$$
\varphi(2, n)=2 \sum_{k=n+1}^{2 n} \frac{1}{k}+\frac{1}{2 n+1}
$$

and

$$
\varphi(4, n)=\sum_{k=n+1}^{2 n} \frac{1}{k}+\sum_{k=n}^{2 n} \frac{1}{2 k+1} .
$$

Thus, the right side of (5.2) may be written as

$$
2 \sum_{k=2 n+1}^{4 n} \frac{1}{k}+\sum_{k=n+1}^{2 n} \frac{1}{k}+\frac{2}{4 n+1}=2 \sum_{k=n+1}^{2 n} \frac{1}{k}+2 \sum_{k=n}^{2 n} \frac{1}{2 k+1}=2 \varphi(4, n),
$$

which completes the proof.

EXAMPLE 4. For each positive integer $n$,

$$
\varphi(4, n)=\frac{1}{2} \sum_{k=n+1}^{2 n} \frac{1}{k}+\sum_{k=2 n+1}^{4 n+1} \frac{1}{k}
$$

Proof. This expression for $\varphi(4, n)$ arises from a rearrangement of the terms in $(5.4)$.

EXAMPLE 5. $\frac{1}{4} \log 3-\frac{1}{3} \log 2=\sum_{k=1}^{\infty} \frac{1}{\{3(2 k-1)\}^{3}-3(2 k-1)}$.

Proof. The right side above is $\frac{1}{2}\{\varphi(3)-\varphi(6)\}$, and so the result follows immediately from the corollaries of Entries 2 and 5.

EXAMPLE 6. $\frac{4}{3} \log 2=1+\sum_{k=1}^{\infty} \frac{2(-1)^{k}}{(3 k)^{3}-3 k}$.

Proof. The right side above is $2 \varphi(6)-\varphi(3)$, and so the result is a consequence of the corollaries to Entries 2 and 5.

EXAMPLE 7. For each positive integer $n$,

$$
2 \varphi(6, n)+\frac{1}{3} \varphi(2, n)=\varphi(3, n)+\varphi(2,3 n)+\frac{2}{(6 n+1)(6 n+2)(6 n+3)}
$$


Proof. By Entries 2 and 5, respectively,

$$
\varphi(3, n)=\sum_{k=n+1}^{3 n+1} \frac{1}{k}
$$

and

$$
\varphi(6, n)=\frac{2}{3} \sum_{k=n+1}^{2 n} \frac{1}{k}+\sum_{k=n}^{3 n} \frac{1}{2 k+1} .
$$

Thus, by (5.3) and (5.7), the left side of (5.5) is equal to

$$
\begin{aligned}
2 \sum_{k=n+1}^{2 n} \frac{1}{k}+2 \sum_{k=n}^{3 n} \frac{1}{2 k+1}+\frac{1}{3(2 n+1)} & =2 \sum_{k=n+1}^{6 n+1} \frac{1}{k}-\sum_{k=n+1}^{3 n} \frac{1}{k}+\frac{1}{6 n+3} \\
& =\sum_{k=n+1}^{3 n} \frac{1}{k}+2 \sum_{k=3 n+1}^{6 n+1} \frac{1}{k}+\frac{1}{6 n+3} \\
& =\sum_{k=n+1}^{3 n+1} \frac{1}{k}+2 \sum_{k=3 n+1}^{6 n} \frac{1}{k}+\frac{36 n^{2}+30 n+8}{(6 n+1)(6 n+2)(6 n+3)} .
\end{aligned}
$$

By (5.3) and (5.6), the far right side of (5.8) is easily seen to be equal to the right side of (5.5).

EXAMPLE 8. $\sum_{k=2}^{13} A\left(\frac{1}{k}\right)=\frac{\pi}{2}+2 A\left(\frac{1}{4}\right)+A\left(\frac{2}{49}\right)+A\left(\frac{3}{232}\right)+A\left(\frac{4}{715}\right)$.

Proof. Apply (3.1) with $n=1$ and with $n=4$. Adding the two results yields the desired equality.

EXAMPLE 9. For each positive integer $n$,

$$
2 \sum_{k=1}^{n+1} A\left(\frac{1}{n+k}\right)=A\left(\frac{n+1}{n}\right)+\sum_{k=1}^{n} A\left(\frac{2 k}{8 k^{4}+2 k^{2}+1}\right)+2 \sum_{k=1}^{n} A\left(\frac{1}{k\left(4 k^{2}+3\right)}\right) \text {. }
$$

Proof. Rewriting the left side of (5.9) and then employing (0.1) and (0.2) several times, we find that

$$
\begin{aligned}
2 \sum_{k=1}^{n+1} A\left(\frac{1}{n+k}\right)= & A(1)+A\left(\frac{1}{2 n+1}\right)+2 \sum_{k=1}^{n}\left\{2 A\left(\frac{1}{2 k}\right)-A\left(\frac{1}{k}\right)\right\} \\
& +\sum_{k=1}^{n}\left\{\left[A\left(\frac{1}{2 k-1}\right)-A\left(\frac{1}{2 k}\right)\right]-\left[A\left(\frac{1}{2 k}\right)-A\left(\frac{1}{2 k+1}\right)\right]\right\} \\
= & A\left(\frac{n+1}{n}\right)+2 \sum_{k=1}^{n}\left\{A\left(\frac{4 k}{4 k^{2}-1}\right)-A\left(\frac{1}{k}\right)\right\} \\
& +\sum_{k=1}^{n}\left\{A\left(\frac{1}{4 k^{2}-2 k+1}\right)-A\left(\frac{1}{4 k^{2}+2 k+1}\right)\right\} \\
= & A\left(\frac{n+1}{n}\right)+2 \sum_{k=1}^{n} A\left(\frac{1}{k\left(4 k^{2}+3\right)}\right)+\sum_{k=1}^{n} A\left(\frac{2 k}{8 k^{4}+2 k^{2}+1}\right) .
\end{aligned}
$$


EXAMPLE 10. For each positive integer $n$,

$$
\begin{aligned}
& \sum_{k=1}^{n} A\left(\frac{1}{n+k}\right)+\sum_{k=0}^{n} A\left(\frac{1}{2 n+2 k+1}\right) \\
& =\frac{\pi}{4}+\sum_{k=1}^{n} A\left(\frac{9 k}{32 k^{4}+22 k^{2}-1}\right)+\sum_{k=1}^{n} A\left(\frac{4 k}{128 k^{4}+8 k^{2}+1}\right) .
\end{aligned}
$$

Proof. We first rewrite the left side of (5.10) and then use (0.1) and (0.2) several times. Accordingly, we get

$$
\begin{array}{rl}
\sum_{k=1}^{n} & A\left(\frac{1}{n+k}\right)+\sum_{k=0}^{n} A\left(\frac{1}{2 n+2 k+1}\right) \\
= & A(1)+\sum_{k=1}^{n}\left\{2 A\left(\frac{1}{4 k}\right)-\left[A\left(\frac{1}{k}\right)-A\left(\frac{1}{2 k}\right)\right]\right\} \\
& +\sum_{k=1}^{n}\left\{\left[A\left(\frac{1}{4 k-1}\right)-A\left(\frac{1}{4 k}\right)\right]-\left[A\left(\frac{1}{4 k}\right)-A\left(\frac{1}{4 k+1}\right)\right]\right\} \\
= & \frac{\pi}{4}+\sum_{k=1}^{n}\left\{A\left(\frac{8 k}{16 k^{2}-1}\right)-A\left(\frac{k}{2 k^{2}+1}\right)\right\}+\sum_{k=1}^{n}\left\{A\left(\frac{1}{16 k^{2}-4 k+1}\right)-A\left(\frac{1}{16 k^{2}+4 k+1}\right)\right\} \\
= & \frac{\pi}{4}+\sum_{k=1}^{n} A\left(\frac{9 k}{32 k^{4}+22 k^{2}-1}\right)+\sum_{k=1}^{n} A\left(\frac{4 k}{128 k^{4}+8 k^{2}+1}\right) .
\end{array}
$$

ENTRY 6. Let $k$ and $n$ be nonnegative integers and define $A_{k}=3^{k}\left(n+\frac{1}{2}\right)-\frac{1}{2}$. Then if $r$ is a positive integer,

$$
\sum_{k=n+1}^{A_{r}} \frac{1}{k}=r+2 \sum_{k=0}^{r-1}(r-k) \sum_{i=A_{k-1}+1}^{A_{k}} \frac{1}{(3 j)^{3}-3 j},
$$

where we define $A_{-1}=0$.

Proof. This proof was given by Ramanujan. First, it is easily shown that $A_{k+1}=$ $3 A_{k}+1, k \geq 0$. Hence, by Entry 2, with $n=A_{k}$,

$$
\sum_{i=A_{k}+1}^{A_{k+1}} \frac{1}{j}=\varphi\left(3, A_{k}\right) \text {. }
$$

Now sum both sides of this equality on $k, 0 \leq k \leq r-1$, to obtain

$$
\sum_{j=n+1}^{A_{i}} \frac{1}{j}=r+2 \sum_{k=0}^{r-1} \sum_{j=1}^{A_{k}} \frac{1}{(3 j)^{3}-3 j} \text {. }
$$

Rearranging the right side above, we deduce the desired equality.

COROLLARY. For each positive integer $r$,

$$
\sum_{k=1}^{(3 r-1) / 2} \frac{1}{k}=r+2 \sum_{k=1}^{r-1}(r-k) \sum_{j=A_{k-1}+1}^{A_{k}} \frac{1}{(3 j)^{3}-3 j},
$$

where $A_{k}=\left(3^{k}-1\right) / 2, k \geq 0$. 
Proof. This corollary is the case $n=0$ of Entry 6.

At this point, Ramanujan claims that if $a_{1}, \ldots, a_{n}$ are in arithmetic progression and if $a_{1}$ and $a_{n}$ are large then $\sum_{k=1}^{n} 1 / a_{k}$ is approximately equal to $2 n /\left(a_{1}+a_{n}\right)$. Unfortunately, this remark is false. For example, consider $S_{n}=\sum_{k=n+1}^{3 n+1} 1 / k$. If Ramanujan were correct, then for large $n, S_{n}$ would approximately be equal to $2(2 n+1) /(4 n+2)=1$. However, by (2.2), $S_{n} \rightarrow \log 3$ as $n \rightarrow \infty$.

Nonetheless, Ramanujan's assertion is correct if $n / a_{1}$ is "small", as we now demonstrate. Letting $a, d$, and $n$ denote positive integers, define

$$
S(a, d, n)=\sum_{k=0}^{n} \frac{1}{a+k d} .
$$

Now, $2 n /\left(a_{1}+a_{n}\right)$ in Ramanujan's notation becomes $2(n+1) /(2 a+n d)$ in our notation. Thus, we wish to examine

$$
\begin{aligned}
S(a, d, n)-\frac{2(n+1)}{2 a+n d}= & \sum_{k=0}^{n} \frac{(n-2 k) d}{(a+k d)(2 a+n d)} \\
= & \frac{d}{2 a+n d}\left\{n\left(\frac{1}{a}-\frac{1}{a+n d}\right)+(n-2)\left(\frac{1}{a+d}-\frac{1}{a+(n-1) d}\right)\right. \\
& \left.+(n-4)\left(\frac{1}{a+2 d}-\frac{1}{a+(n-2) d}\right)+\ldots+T_{n}\right\},
\end{aligned}
$$

where

$$
T_{n}= \begin{cases}\frac{1}{a+(n-1) d / 2}-\frac{1}{a+(n+1) d / 2}, & \text { if } n \text { is odd, } \\ \frac{2}{a+(n-2) d / 2}-\frac{2}{a+(n+2) d / 2}, & \text { if } n \text { is even. }\end{cases}
$$

Hence, with $d$ fixed,

$$
S(a, d, n)-\frac{2(n+1)}{2 a+n d}=O\left\{\frac{n^{2}}{a}\left(\frac{1}{a}-\frac{1}{a+n d}\right)\right\}=O\left\{\left(\frac{n}{a}\right)^{3}\right\},
$$

as $n / a$ tends to 0 . Thus, under this assumption, Ramanujan's approximation is, indeed, valid.

EXAMPLE 1. $\sum_{k=1}^{13} \frac{1}{k}=3+\frac{1}{6}+\frac{1}{105}+\frac{1}{360}+\frac{1}{858}$.

Proof. In the previous corollary, let $r=3$. Since $A_{0}=0, A_{1}=1, A_{2}=4$, and $A_{3}=13$, we find that

$$
\sum_{k=1}^{13} \frac{1}{k}=3+\frac{4}{3^{3}-3}+2\left(\frac{1}{6^{3}-6}+\frac{1}{9^{3}-9}+\frac{1}{(12)^{3}-12}\right)
$$

and the result follows. 
EXAMPLE 2. $H \equiv \sum_{k=1}^{1000} \frac{1}{k}=7 \frac{1}{2}$ "very nearly."

Proof. In the previous corollary, let $r=7$. In addition to $A_{0}, A_{1}, A_{2}$, and $A_{3}$ calculated above, we need the values $A_{4}=40, A_{5}=121, A_{6}=364$, and $A_{7}=1093$. Thus,

$$
\begin{aligned}
\sum_{k=1}^{1093} \frac{1}{k}= & 7+\frac{1}{2}+5\left(\frac{1}{105}+\frac{1}{360}+\frac{1}{858}\right)+8 \sum_{k=5}^{13} \frac{1}{(3 k)^{3}-3 k}+6 \sum_{k=14}^{40} \frac{1}{(3 k)^{3}-3 k} \\
& +4 \sum_{k=41}^{121} \frac{1}{(3 k)^{3}-3 k}+2 \sum_{k=122}^{364} \frac{1}{(3 k)^{3}-3 k} \\
= & 7+0.5+0.067335442 \ldots+0.006435448 \ldots \\
& +0.000541282 \ldots+0.000040137 \ldots+0.000002230 \ldots \\
= & 7.574354539 \ldots
\end{aligned}
$$

Next, by the remarks prior to Example 1 ,

$$
\sum_{k=1001}^{1093} \frac{1}{k} \approx \frac{186}{2094}=0 \cdot 088825214 \ldots
$$

Thus, from (6.1) and (6.2) we conclude that $H \approx 7.48552932 \ldots$ This is probably the method that Ramanujan employed to estimate $H$. On the other hand, by using the Euler-Maclaurin summation formula or a programmable calculator, it can readily be shown that $H=7 \cdot 48547086 \ldots$ In any event, the estimate of $7 \frac{1}{2}$ for $H$ is not as good as Ramanujan would lead us to believe.

ENTRY 7. Let $n>0$ and suppose that $r$ is a natural number. Then

$$
\sum_{k=0}^{r-1} A\left(\frac{2}{(n+2 k+1)^{2}}\right)=A\left(\frac{2 r}{n^{2}+2 n r+1}\right) \text {. }
$$

Proof. The proof is very briefly sketched by Ramanujan. Since $n>0$, it follows from (0.2) that

$$
A\left(\frac{1}{n+2 k}\right)-A\left(\frac{1}{n+2 k+2}\right)=A\left(\frac{2}{(n+2 k+1)^{2}}\right) \text {. }
$$

Now sum both sides of (7.1) on $k, 0 \leq k \leq r-1$, to get

$$
A\left(\frac{1}{n}\right)-A\left(\frac{1}{n+2 r}\right)=\sum_{k=0}^{r-1} A\left(\frac{2}{(n+2 k+1)^{2}}\right) .
$$

An application of (0.2) on the left side of (7.2) completes the proof.

COROLLARY. For $n>0$,

$$
\sum_{k=0}^{\infty} A\left(\frac{2}{(n+2 k+1)^{2}}\right)=A\left(\frac{1}{n}\right)
$$


Proof. Ler $r$ tend to $\infty$ in (7.2).

EXAMPLE 1. For $n>0$,

$$
\sum_{k=1}^{\infty} A\left(\frac{2}{(n+k)^{2}}\right)=A\left(\frac{2 n+1}{n^{2}+n-1}\right)+\rho(n),
$$

where $\rho(n)=\pi$ if $n<(\sqrt{5}-1) / 2$ and $\rho(n)=0$ otherwise.

Proof. The proof is sketched by Ramanujan. From the previous corollary and (0.1),

$$
\begin{aligned}
\sum_{k=1}^{\infty} A\left(\frac{2}{(n+k)^{2}}\right) & =\sum_{k=0}^{\infty} A\left(\frac{2}{(n+2 k+1)^{2}}\right)+\sum_{k=0}^{\infty} A\left(\frac{2}{(n+2 k+2)^{2}}\right) \\
& =A\left(\frac{1}{n}\right)+A\left(\frac{1}{n+1}\right) \\
& =A\left(\frac{2 n+1}{n^{2}+n-1}\right)+\rho(n),
\end{aligned}
$$

since $\rho(n)=\pi$ if and only if $n^{2}+n<1$, i.e., $n<(\sqrt{5}-1) / 2$. This completes the proof.

EXAMPLE 2. For $n>0$,

$$
\sum_{k=1}^{\infty}(-1)^{k+1} A\left(\frac{2}{(n+k)^{2}}\right)=A\left(\frac{1}{n^{2}+n+1}\right) .
$$

Proof. The proof is very similar to that of Example 1.

EXAMPLE 3. For $n>0$,

$$
\sum_{k=1}^{\infty} A\left(\frac{1}{2(n+k)^{2}}\right)=A\left(\frac{1}{2 n+1}\right) .
$$

Proof. Replace $n$ by $2 n+1$ in the corollary to Entry 7 .

EXAMPLE 4. $\sum_{k=1}^{\infty} A\left(\frac{2}{k^{2}}\right)=\frac{3 \pi}{4}$.

Proof. Since the series on the left side of (7.4) converges uniformly for $0 \leq n \leq 1$, we may let $n$ tend to 0 on both sides of (7.4). The desired result then immediately follows.

Example 4 and the first equality in Example 5 which follows were apparently first established by Glaisher [7] in 1878. This paper contains many other examples of this sort. Example 4 is a problem in Chrystal's book [6, p. 357] as well as in Loney's textbook $[13$, p. 206]. The latter fact is interesting because the borrowing of Loney's book from a friend while in fourth form was evidently a pivotal event in Ramanujan's mathematical development [18, p. xii]. Still another proof of Example 4 can be found in Wheelon's book [21, p. 46]. 
EXAMPLE 5. $\sum_{k=1}^{\infty} A\left(\frac{1}{2 k^{2}}\right)=\frac{\pi}{4}=\sum_{k=1}^{\infty}(-1)^{k+1} A\left(\frac{2}{k^{2}}\right)$.

Proof. The series on the left sides of (7.5) and (7.6) each converge uniformly for $0 \leq n \leq 1$. Letting $n$ tend to 0 in (7.5) and (7.6), we immediately deduce the evaluations above.

EXAMPLE 6. $\sum_{k=1}^{\infty} A\left(\frac{1}{(1+\sqrt{2} k)^{2}}\right)=\frac{\pi}{8}$.

Proof. In Example 3 let $n=1 / \sqrt{2}$. A short calculation shows that $\tan (\pi / 8)=$ $1 /(\sqrt{2}+1)$, and so the result readily follows.

$$
\text { EXAMPLE 7. } \sum_{k=1}^{\infty} A\left(\frac{8}{(2 k-1+\sqrt{5})^{2}}\right)=\frac{\pi}{2} \text {. }
$$

Proof. Since the left side of (7.4) converges uniformly for $0 \leq n \leq 1$, we may let $n$ tend to $(\sqrt{5}-1) / 2+0$. The desired equality then follows.

EXAMPLE 8. $\sum_{k=0}^{\infty} A\left(\frac{2}{(2 k+1)^{2}}\right)=\frac{\pi}{2}$.

Proof. The series on the left side of (7.3) converges uniformly for $0 \leq n \leq 1$. Letting $n$ tend to 0 in (7.3) yields the desired result.

Example 8 is also found in Glaisher's paper [7].

In Entry 8 Ramanujan considers an entire function $f$ with zeros $z_{1}, z_{2}, \ldots \mathrm{He}$ evidently assumes that $\sum_{k=1}^{\infty} 1 /\left|z_{k}\right|$ converges and states a corresponding special case of the Hadamard factorization theorem [20, p. 250]. He then takes the logarithmic derivative of this product representation for $f$ and evaluates it at $z=0$.

In Entries 9(i) and (ii) the familiar product representations for $\sin x$ and $\cos x$ are stated. Corollaries 1 and 2 of Entry 9 give the well-known product representations of $\sinh x$ and $\cosh x$.

COROLLARY 3. For each complex number $x$,

$$
\cos (x / 4)+\sin (x / 4)=\prod_{k=0}^{\infty}\left(1+\frac{(-1)^{k} x}{(2 k+1) \pi}\right)
$$

COROLLARY 4. Let $x$ and a be complex, where $a$ is not an integral multiple of $\pi$. Then

$$
\frac{\sin (x+a)}{\sin a}=\frac{x+a}{a} \prod_{k=1}^{\infty}\left\{\left(1-\frac{x}{k \pi-a}\right)\left(1+\frac{x}{k \pi+a}\right)\right\} \text {. }
$$

Corollary 3 is easily derived from Corollary 4 by setting $a=\pi / 4$ and replacing $x$ by $x / 4$. Corollaries 3 and 4 are rather straightforward exercises which can be found in Bromwich's book [5, p. 224], for example, and so it is pointless to give a proof of Corollary 4 here. 
EXAMPLE 1. Let $x$ and $a$ be complex, where $a$ is not an odd multiple of $\pi / 2$. Then

$$
\frac{\cos (x+a)}{\cos a}=\prod_{k=1}^{\infty}\left\{\left(1-\frac{x}{\left(k-\frac{1}{2}\right) \pi-a}\right)\left(1+\frac{x}{\left(k-\frac{1}{2}\right) \pi+a}\right)\right\} .
$$

Example 1 is easily derived from Corollary 4 by replacing $a$ by $a+\pi / 2$. Example 2 below follows from Corollary 4 and Example 1 upon the use of the identity

$$
1+\frac{\sin x}{\sin a}=\frac{\sin \left\{\frac{1}{2}(x+a)\right\}}{\sin \left(\frac{1}{2} a\right)} \frac{\cos \left\{\frac{1}{2}(x-a)\right\}}{\cos \left(\frac{1}{2} a\right)} .
$$

EXAMPLE 2. Let $x$ and $a$ be complex, where $a$ is not an integral multiple of $\pi$. Then $1+\frac{\sin x}{\sin a}=\frac{a+x}{a} \prod_{k=1}^{\infty}\left\{\left(1-\frac{x}{2 k \pi-a}\right)\left(1+\frac{x}{2 k \pi+a}\right)\left(1-\frac{x}{(2 k-1) \pi+a}\right)\left(1+\frac{x}{(2 k-1) \pi-a}\right)\right\}$.

Next, Ramanujan asserts that if the value of $F(x)=\prod_{k=1}^{\infty}\left(1+a_{k} x\right)$ is known, then it is possible to find the value of $\prod_{k=1}^{\infty}\left(1+a_{k}^{n} x^{n}\right)$, where $n$ is a positive integer. Ramanujan's declaration evidently arises from the identity

$$
\prod_{k=1}^{\infty}\left(1+a_{k}^{n} x^{n}\right)=\prod_{j=1}^{n} F\left(-\omega^{2 j-1} x\right)
$$

which is a consequence of the factorization

$$
1+a^{n} x^{n}=\prod_{j=1}^{n}\left(1-a \omega^{2 j-1} x\right)
$$

where $\omega=\exp (\pi i / n)$.

In Entry 10, the familiar partial fraction decompositions of $\cot x, \tan x, \csc x$, and $\sec x$ are given [5, pp. 217, 225].

ENTRY 11. Let $x$ and $a$ be real. Then

$$
A\left(\frac{x}{a}\right)+\sum_{k=1}^{\infty}\left\{A\left(\frac{x}{k \pi+a}\right)-A\left(\frac{x}{k \pi-a}\right)\right\}=A(\tanh x \cot a) .
$$

Proof. The main idea for the proof is indicated by Ramanujan. By Corollary 4 of Entry 9 ,

$$
\begin{aligned}
\operatorname{Im} \log \left(\frac{\sin (a+i x)}{\sin a}\right) & =\operatorname{Im} \log \left\{\left(1+\frac{i x}{a}\right) \prod_{k=1}^{\infty}\left(1-\frac{i x}{k \pi-a}\right)\left(1+\frac{i x}{k \pi+a}\right)\right\} \\
& =A\left(\frac{x}{a}\right)+\sum_{k=1}^{\infty}\left\{A\left(\frac{x}{k \pi+a}\right)-A\left(\frac{x}{k \pi-a}\right)\right\},
\end{aligned}
$$


up to an additive multiple of $\pi$. On the other hand,

$$
\begin{aligned}
\operatorname{Im} \log \left(\frac{\sin (a+i x)}{\sin a}\right) & =\operatorname{Im} \log (\cosh x+i \sinh x \cot a) \\
& =A(\tanh x \cot a),
\end{aligned}
$$

up to an additive multiple of $\pi$. Combining (11.2) and (11.3), we have shown that (11.1) is valid up to an additive multiple of $\pi$. We now show that this additive multiple of $\pi$ is, indeed, 0.

First, if $a$ is a multiple of $\pi$, it is readily checked that (11.1) is valid. Suppose now that $a$ is fixed but not a multiple of $\pi$. For $x=0,(11.1)$ is certainly true. Since both sides of (11.1) are continuous functions of $x$, that additive multiple of $\pi$ must be 0 for all $x$. Since $a$ is arbitrary, the proof is complete.

COROLLARY 1. Let $x$ and $a$ be real. Then

$$
\sum_{k=-\infty}^{\infty}(-1)^{k} A\left(\frac{x}{k \pi+a}\right)=A(\sinh x \csc a) \text {. }
$$

Proof. First, by Example 2 of Entry 9,

$$
\begin{aligned}
& A(\sinh x \csc a)=\operatorname{Im} \log \left(1+i \frac{\sinh x}{\sin a}\right) \\
& =\operatorname{Im} \log \left\{\left(1+\frac{i x}{a}\right) \prod_{k=1}^{\infty}\left(1-\frac{i x}{2 k \pi-a}\right)\left(1+\frac{i x}{2 k \pi+a}\right)\left(1-\frac{i x}{(2 k-1) \pi+a}\right)\left(1+\frac{i x}{(2 k-1) \pi-a}\right)\right\} \\
& =\sum_{k=-\infty}^{\infty}(-1)^{k} A\left(\frac{x}{k \pi+a}\right),
\end{aligned}
$$

up to an additive multiple of $\pi$. Thus, (11.4) is valid up to an additive multiple of $\pi$. To show that this multiple of $\pi$ is 0 , we proceed in the same manner as in the proof of Entry 11.

COROLLARY 2. For real $x$,

$$
\sum_{k=0}^{\infty}(-1)^{k} A\left(\frac{x}{2 k+1}\right)=A(\tanh (\pi x / 4))
$$

Proof. Replacing $x$ by $\pi x / 4$ and setting $a=\pi / 4$ in Entry 11 , we readily achieve the desired formula.

COROLlaRY 3. For real $x$,

$$
\sum_{k=-\infty}^{\infty}(-1)^{k} A\left(\frac{x}{4 k+1}\right)=A(\sqrt{2} \sinh (\pi x / 4)) \text {. }
$$

Proof. In Corollary 1 of Entry 11 replace $x$ by $\pi x / 4$ and let $a=\pi / 4$ to deduce the formula sought. 
The next two examples are obtained by replacing $a$ by $\pi / 2-a$ in Entry 11 and Corollary 1, respectively. In the second notebook there is a minor misprint in Example 1.

EXAMPLE 1. For $x$ and $a$ real,

$$
\sum_{k=1}^{\infty}\left\{A\left(\frac{x}{\frac{1}{2}(2 k-1) \pi-a}\right)-A\left(\frac{x}{\frac{1}{2}(2 k-1) \pi+a}\right)\right\}=A(\tanh x \tan a) .
$$

EXAMPLE 2. For $x$ and $a$ real,

$$
\sum_{k=-\infty}^{\infty}(-1)^{k} A\left(\frac{x}{\frac{1}{2}(2 k+1) \pi-a}\right)=A(\sinh x \sec a) .
$$

Entry 11 is an exercise in both the books of Chrystal [6, p. 373] and Loney [13, p. 208]. Corollary 2 is also a problem in Loney's book [13]. Entry 11, Corollaries 1 and 2, and Example 1 are given in Hansen's tables [10, p. 276]. Several other arctangent series in the spirit of those given above are summed in this compendium [10]. Ramanujan himself summed other arctangent series in [14], [18, p. 42]. Glasser and Klamkin [8] have summed several arctangent series in an elementary fashion. Further examples of arctangent series are found in Bromwich's book [5, pp. 314-315].

EXAMPLE 3. $\prod_{k=1}^{\infty}\left(1+1 / k^{3}\right)=\frac{1}{\pi} \cosh (\pi \sqrt{3} / 2)$.

EXAMPLE 4. $\prod_{k=2}^{\infty}\left(1-1 / k^{3}\right)=\frac{1}{3 \pi} \cosh (\pi \sqrt{3} / 2)$.

Examples 3 and 4 constitute the second problem that Ramanujan submitted to the Journal of the Indian Mathematicial Society [17], [18, p. 322]. In a later paper [15], [18, pp. 50-52], Ramanujan studied the more general product $\prod_{k=0}^{\infty}\left\{1+\left(\frac{x}{a+k d}\right)^{3}\right\}$.

ENTRY 12. Ramanujan here presents a method for approximating the root $z_{0}$ of smallest modulus of the equation

$$
\sum_{k=1}^{\infty} A_{k} z^{k}=1
$$

It is assumed that all other roots of $(12.1)$ have moduli strictly greater than $\left|z_{0}\right|$. For $|z|$ sufficiently small, write

$$
\frac{1}{1-\sum_{k=1}^{\infty} A_{k} z^{k}}=\sum_{k=1}^{\infty} P_{k} z^{k-1}
$$

It follows easily that $P_{1}=1$ and

$$
P_{n}=\sum_{j=1}^{n-1} A_{j} P_{n-j}, \quad(n \geq 2) .
$$


Now assume that $\lim _{n \rightarrow \infty} P_{n} / P_{n+1}$ exists and is equal to $L$. Then, of course, the radius of convergence of $\sum_{k=1}^{\infty} P_{k} z^{k}$ is equal to $|L|$. Moreover, by a theorem of Fabry [9, pp. 39-40], $L$ is a singularity of the function represented by this series. It follows that if the radius of convergence of the series $\sum_{k=1}^{\infty} A_{k} z^{k}$ is greater then $|L|$, then $z=L$ is a root of (12.1). Ramanujan's discourse is characteristically brief; he gives (12.2) and claims, with no hypotheses, that $P_{n} / P_{n+1}$ approaches a root of (12.1).

In the case that (12.1) is an algebraic equation, this method is originally due to Daniel Bernoulli. Accounts of Bernoulli's method may be found in the books of Whittaker and Robinson.[22, pp. 98-99] and Henrichi [11, p. 663]. Usually a change of variable is made so that the method yields the approximate value of the root with largest modulus. Bernoulli's method has been generalized by Aitken [1] who found a way to approximate any root of a polynomial.

Ramanujan concludes this chapter by giving six examples to illustrate his method. $\mathrm{He}$ takes $P_{0}=0$, and so the first convergent is always $0 / 1$.

EXAMPLE 1. The roots of $x+x^{2}=1$ are $(-1 \pm \sqrt{5}) / 2$, and so $(\sqrt{5}-1) / 2=0 \cdot 618034 \ldots$ is the root of least modulus. Ramanujan gives the first eight convergents to this root with the last being $P_{7} / P_{8}=13 / 21=0.619048 \ldots$

EXAMPLE 2. By Newton's method the real root of $x+x^{2}+x^{3}=1$ is $0 \cdot 543689013 \ldots$ Ramanujan gives the first eight convergents to this root with the last equal to $24 / 44=$ $0 \cdot 5454 \ldots$.

EXAMPLE 3. Ramanujan lists the first ten convergents to the real root of $x+x^{3}=1$, with the last convergent being $13 / 19=0.684210 \ldots$ By Newton's method, this root is $0 \cdot 682327804 \ldots$

EXAMPLE 4. The last polynomial equation examined by Ramanujan is $2 x+x^{2}+x^{3}=$ 1. He calculates seven convergents to the real root and finds the seventh to be $84 / 214=$ $0 \cdot 392523 \ldots$ This root is $0 \cdot 392646782 \ldots$, by Newton's method.

At this point, Ramanujan claims that "If $p / q$ and $r / s$ are two consecutive convergents to $x$, then we may take $(m p+n r) /(m q+n s)$ in a suitable manner equivalent to $x$." If $m$ and $n$ are to be taken as real, then Ramanujan's remark is pointless, for then this ratio may be made to take any real value. On the other hand, if $m$ and $n$ are to be understood as positive, then Ramanujan's assertion is false. Ramanujan's claim would be valid if the limit $L$ were always between two consecutive convergents. However, this may not be true. For example, the last three convergents $13 / 33,33 / 84$, and $84 / 214$ given by Ramanujan in Example 4 satisfy the inequalities $13 / 33>33 / 84>84 / 214$.

EXAMPLE 1. In this example Ramanujan examines $e^{x}=2$ and finds the first six convergents to $\log 2=0.69315 \ldots$ The sixth convergent is $375 / 541=0.69316 \ldots$ 
EXAMPLE 2. In this last example Ramanujan approximates the root of $e^{-x}=x$. He calculates five convergents with the last one equal to $148 / 261=0.567049 \ldots$ By Newton's method, the root is $0.567143290 \ldots$.

E. M. Wright has written several papers [23], [24], [25], [26], [27], in which he has studied solutions of equations generalizing the one in the last example. Such equations are very important in the theory of differential-difference equations.

\section{REFERENCES}

1. A. C. Aitken, On Bernoulli's numerical solution of algebaic equations, Proc. Roy. Soc. Edinburgh, Sect. A 46 (1926), 289-305.

2. R. Ayoub, An introduction to the analytic theory of numbers, (American Mathematical Society, Providence, 1963).

3. B. C. Berndt, Ramanujan's notebooks, Math. Mag. 51 (1978), 147-164.

4. B. C. Berndt, Chapter 14 of Ramanujan's second notebook, Enseignement Math. 26 (1980), $1-65$.

5. T. J. I'A. Bromwich, An introduction to the theory of infinite series, second ed., (Macmillan, London, 1926).

6. G. Chrystal, Algebra, Part II, second ed., (A. and C. Black, London, 1922).

7. J. W. L. Glaisher, A theorem in trigonometry, Quart. J. Math. Oxford 15 (1878), 151-157.

8. M. L. Glasser and M. S. Klamkin, On some inverse tangent summations, Fibonacci Quart. 14 (1976), 385-388.

9. J. S. Hadamard et M. Mandelbrojt, La Série de Taylor, (Gauthier-Villars, Paris, 1926).

10. E. R. Hansen, A table of series and products, (Prentice-Hall, Englewood Cliffs, 1975).

11. P. Henrichi, Applied and computational complex analysis, Vol. 1, (John Wiley \& Sons, New York, 1974).

12. M. Lionnet, Question 1294, solution by M. A. Laisant, Nouv. Ann. Math., Ser. 2, 18 (1879), 330-332.

13. S. L. Loney, Plane trigonometry, Part II, (University Press, Cambridge, 1952).

14. S. Ramanujan, On the integral $\int_{0}^{x} \frac{\tan ^{-1} t}{t} d t$, J. Indian Math. Soc. 7 (1915), 93-96.

15. S. Ramanujan, On the product $\prod_{n=0}^{n=\infty}\left[1+\left(\frac{x}{a+n d}\right)^{3}\right]$, J. Indian Math. Soc. 7 (1915), 209-211.

16. S. Ramanujan, Question 260, J. Indian Math. Soc. 3 (1911), 43.

17. S. Ramanujan, Question 261, J. Indian Math. Soc. 3 (1911), 43.

18. S. Ramanujan, Collected papers, (Chelsea, New York, 1962).

19. S. Ramanujan, Notebooks (2 volumes), (Tata Institute of Fundamental Research, Bombay, 1957).

20. E. C. Titchmarsh, The theory of functions, second ed., (Oxford University Press, London, 1939).

21. A. D. Wheelon, Tables of summable series and integrals involving Bessel functions, (Holden-Day, San Francisco, 1968).

22. E. T. Whittaker and G. Robinson, The calculus of observations, second ed., (Blackie and Son, Glasgow, 1926).

23. E. M. Wright, Solution of the equation $z e^{z}=a$, Bull. Amer. Math. Soc. 65 (1959), 89-93.

24. E. M. Wright, Solution of the equation $z e^{z}=a$, Proc. Roy. Soc. Edinburgh, Sect. A 65 (1959), 192-203. 
25. E. M. Wright, Solution of the equation $(p z+q) e^{z}=r z+s$, Bull. Amer. Math. Soc. 66 (1960), 277-281.

26. E. M. Wright, Solution of the equation $(z+b) e^{z+a}= \pm(z+b)$, Proc. Roy. Soc. Edinburgh, Sect. A 65 (1960/61), 358-371.

27. E. M. Wright, Stability criteria and the real roots of a transcendental equation, SIAM J. Appl. Math. 9 (1961), 136-148.

Bruce C. Berndt

Department of Mathematics

UNIVERSITY OF ILLINOIS

URBANA, IlLINOIS 61801

U.S.A.
Padmini T. Joshi

Department of Mathematics Ball State University MunCIE, Indiana 47306 U.S.A. 